\title{
Decoupling of the longitudinal polarization of the vector field in the massless Higgs-Kibble model.
}

\author{
A.A.Slavnov * \\ Steklov Mathematical Institute, Russian Academy of Sciences \\ Gubkina st.8, GSP-1,119991, Moscow
}

September 13, 2018

\begin{abstract}
It is shown that the three dimensionally longitudinal component of the vector field decouples in the massless limit of nonabelian Higgs model.
\end{abstract}

\section{Introduction.}

It was known long ago that the massless limit of the global $U(1)$ invariant theory of massive neutral vector field interacting with the matter fields reproduces the results of quantum electrodynamics (QED). In the limit $\mu \rightarrow 0$ the three dimensionally longitudinal component of the vector field decouples of all other excitations and we are left with two threedimensionally transversal polarizations of the photon interacting with matter fields.

In the nonabelian case the situation is different. In the limit $\mu \rightarrow 0$ the massive Yang-Mills model the three dimensionally longitudinal polarization of the vector field does not decouple [1]. At one loop it gives a finite contribution and at higher loops in the limit $\mu \rightarrow 0$ we have pole singularities c. It is natural to suggest that the massless limit can be achieved in the Higgs model, leading to the massless Yang-Mills field interacting with the massless Higgs meson.

Recently in the paper [2] it was stated that in the limit $\mu \rightarrow 0$ of the nonabelian Higgs model the longitudinal component of the vector field does not decouple, but undergoes the metamorphosis to the massless scalar fields, corresponding to the Goldstone bosons of the Higgs model.

In this paper we argue that the hypothesis about decoupling of the longitudinal component of the vector field in the massless limit of the Higgs-Kibble model is correct. The statement of the paper [2] corresponds to another massless limit: massless

*E-mail: slavnov@mi.ras.ru 
Yang-Mills field interacting with the complex scalar doublet with real nonzero mass, when this mass vanishes. Although if the mass of the Yang-Mills field is less than the accuracy of measurement both these scenarios correspond in the hypothetical limit $\mu \rightarrow 0$ to the massless Yang-Mills theory, the number of physical degrees of freedom is different and these two scenarios may be distinguished experimentally.

\section{Decoupling of the longitudinal component of the vector field in the Higgs-Kibble model in the limit $\mu=0$.}

The Higgs-Kibble model is gauge invariant and may be considered in different gauges. In the unitary gauge $\varphi^{a}=0$, where

$$
\varphi=\left(\frac{i \varphi_{1}+\varphi_{2}}{\sqrt{2}}, \quad \frac{\sigma-i \varphi_{3}}{\sqrt{2}}\right)
$$

the Lagrangian has a form

$$
\begin{array}{r}
L=-\frac{1}{4} F_{\mu \nu}^{a} F_{\mu \nu}^{a}+\frac{m_{1}^{2}}{2} A_{\mu}^{a} A_{\mu}^{a}+\frac{1}{2} \partial_{\mu} \sigma \partial_{\mu} \sigma-\frac{m_{2}^{2}}{2} \sigma^{2} \\
+\frac{m_{1} g}{2} \sigma A_{\mu}^{2}+\frac{g^{2}}{8} \sigma^{2} A_{\mu}^{2}-\frac{g m_{2}^{2}}{4 m_{1}} \sigma^{3}-\frac{g^{2} m_{2}^{2}}{32 m_{1}^{2}} \sigma^{4} \\
m_{1}=\frac{\mu g}{\sqrt{2}} ; \quad m_{2}=2 \lambda \mu
\end{array}
$$

In this gauge the spectrum of the model is obvious: nine massive excitations corresponding to massive vector field $A_{\mu}^{a}$, and one scalar field $\sigma$. Goldstone bosons $\varphi^{a}$ and dynamical ghost fields $c, \bar{c}$ are absent.

It is known that although this gauge is not manifestly renormalizable, for any invariant regularization all nonrenormalizable divergencies present in observable gauge invariant amplitudes cancel and to calculate a renormalized scattering amplitude it is sufficient to redefine the parameters entering the Lagrangian (2): masses, charge and wave function normalization of the fields.

We shall be interested in the amplitudes for the massless fields, when the parameter $\mu=0$. These amplitudes are infected by the infrared divergencies, but we shall ignore these logarithmic divergencies, assuming that $\mu$ is small but finite. These divergencies are absent in the gauge invariant Green functions, whose value after renormalization also does not depend on a gauge.

To avoid a necessity to look for cancelation of nonrenormalizable divergencies in observable amplitudes we shall use not unitary, but the Lorentz gauge $\partial_{\mu} A_{\mu}^{a}=0$. The corresponding effective Lagrangian looks as follows

$$
\begin{array}{r}
L_{e f}=-\frac{1}{4} F_{\mu \nu}^{a} F_{\mu \nu}^{a}+\left(D_{\mu} \varphi-\hat{\mu}\right)^{*}\left(D_{\mu} \varphi-\hat{\mu}\right)-\kappa\left[\left(\varphi^{*}-\hat{\mu}\right)(\varphi-\hat{\mu})-\frac{\mu^{2}}{g^{2}}\right]^{2} \\
+\lambda^{a} \partial_{\mu} A_{\mu}^{a}+i \bar{c}^{a} \partial_{\mu} D_{\mu} c^{a} ; \quad \hat{\mu}=\left(0, \frac{\mu \sqrt{2}}{g}\right) .
\end{array}
$$


Here $\bar{c}^{a}$ and $c^{a}$ are Faddeev-Popov ghosts described by the anticommuting scalar fields.

The spectrum of the theory in this gauge includes apart from physical quanta also unphysical component of the vector field, Faddeev-Popov ghosts and Goldstone bosons $\varphi^{a}$.

The Lagrangian (3) is invariant with respect to the BRST transformations

$$
\begin{array}{r}
\delta A_{\mu}^{a}=\left(D_{\mu} c\right)^{a} \\
\delta \varphi^{a}=\mu c^{a}+\frac{g}{2} \varepsilon^{a b d} \varphi^{b} c^{d}+\frac{g}{2} \sigma c^{a} \\
\delta \bar{c}^{a}=\lambda^{a} \\
\delta c^{a}=g \varepsilon^{a b d} c^{b} c^{d} \\
\delta \sigma=-\frac{g}{2} \varphi^{a} c^{a} \\
\delta \lambda^{a}=0 .
\end{array}
$$

By the Noether theorem this invariance generates the conserved charge $Q$, and physical asymptotic states are selected by the condition

$$
Q^{0} \mid \varphi>_{a s}=0
$$

where $Q^{0}$ is the asymptotic BRST charge.

For any $\mu$ different from zero the invariance of the Lagrangian (3) with respect to the transformations (4) leads to decoupling of the nine physical vector field excitations and the excitation corresponding to the scalar field $\sigma$ from the excitations corresponding to unphysical components of the vector field, Faddeev-Popov ghosts and the Goldstone bosons $\varphi^{a}[3]$.

The field $A_{\mu}^{a}$ may be decomposed into the components with different polarizations

$$
A_{i}^{a}(\mathbf{k})=e_{i}^{1} a_{a}^{1}+e_{i}^{2} a_{2}^{a}+\frac{k_{i} k_{0}}{|k| m_{1}} a_{3}^{a} ; \quad A_{0}^{a}(\mathbf{k})=\frac{|k|}{m_{1}} a_{3}^{a}
$$

In the eq.(6) the vectors $e_{1}$ and $e_{2}$ are the unit vectors orthogonal to each other and to the momentum $\mathbf{k}$.

Having in mind that the propagator of the vector field in the transversal gauge looks as follows

$$
D_{a b}^{\mu \nu}=\delta_{a b} \frac{g^{\mu \nu}-k^{\mu} k^{\nu} k^{-2}}{k^{2}-m^{2}}
$$

we see that the amplitude, describing the transition from the transversally polarized state to another transversally polarized state is finite in the limit $\mu=0$. On the other hand the matrix elements between states including the longitudinal components of the vector field in general are singular in the limit $\mu \rightarrow 0$. Nevertheless, as we shall show, in the limit $\mu \rightarrow 0$ the longitudinal states decouple and the scattering matrix is unitary in the space including only three dimensionally transversal polarizations and the massless Higgs scalar.

Let us consider the forward scattering between states which do not include longitudinal states. As follows from the discussion above, we may define the unitary 
scattering matrix by using the projectors to the states including only the three polarizations of the massive vector field and the massive Higgs meson: $\tilde{S}=P S P$. The matrix element describing the forward scattering of the transversal polarizations of the vector field and the Higgs mesons has a definite limit when $\mu \rightarrow 0$. By the optical theorem the imaginary part of the amplitude of the forward scattering is proportional to the total cross section of the process. That means that the sum

$$
\sum_{l}|<n| \tilde{S}|l>|^{2} \sim \operatorname{Im}<n|\tilde{S}| n>
$$

where the vectors $\mid n>$ do not include the longitudinal polarizations, and the vectors $\mid l>$ span the complete space where the scattering matrix acts. The r.h.s. of the equation (8) has a limit when $\mu \rightarrow 0$ and this limit coincides with the imaginary part of the corresponding amplitude in the massless theory. This theory is known to be unitary in the space including only transversal polarizations of the vector field and massless Higgs meson. As all the vectors including only physical components of the vector field and the Higgs field have positive definite norms we conclude that the amplitudes $\langle n|\tilde{S}| m\rangle=0$ if the vector $\mid m>$ contains at least one longitudinally polarized quant. That means that as it happens in the case of neutral vector meson longitudinal polarizations of the massive Yang-Mills field decouple in the limit $\mu \rightarrow$ 0 , and the resulting theory describes in this limit the massless Yang-Mills field interacting with the massless Higgs bosons.

It is worth to mention that the same result may be obtained starting from the unitary gauge. One should remember however that in the unitary gauge the variables $A_{0}^{a}$ are not dynamical and should be excluded by solving the constraint equation before the quantization.

Namely, the equation of motion for $A_{0}$ does not contain time derivative of canonical variables

$$
\left(m_{1}+\frac{g \sigma}{2}\right)^{2} A_{0}=D_{k} F_{0 k} ; \quad F_{0 k}=P_{k} .
$$

Solving this equation and substituting the solution to the Lagrangian (2) we get the theory described by the nondegenerate Lagrangian, which may be quantized in a usual way. The equation (9) is singular in the limit $\mu \rightarrow 0$ and one should look for the compensation of the singularities generated by the solution of the eq.(9) and the singularities generated by the decomposition of the field $A_{\mu}$ to the states with different polarizations. Using the Lorentz gauge we avoid this complicated procedure.

A massless Yang-Mills field interacting with the massless scalar may be also described by the other model. Let us consider the theory of the Yang-Mills field with zero mass interacting with the scalar complex doublet with a real mass. This theory is also BRST invariant, but in this case the scalar fields $\varphi^{a}$ do not contribute to the asymptotic BRST charge, which has a form

$$
\hat{Q}_{B}^{0}=\int d^{3} k\left[\left(a_{0}^{+}+a_{3}^{+}\right) c^{-}+c^{+}\left(a_{0}^{-}+a_{3}^{-}\right)\right]
$$

As usual one may introduce the operator

$$
\hat{K}=\int d^{3} k\left[\left(a_{0}^{+}-a_{3}^{+}\right) \bar{c}^{-}+\bar{c}^{+}\left(a_{0}^{-}-a_{3}^{-}\right)\right]
$$


The number operator for the unphysical states may be presented as anticommutator of the operators $\hat{Q}_{0}$ and $\hat{K}$

$$
\hat{N}_{\text {unph }}=\left[\hat{Q}_{0}, \hat{K}\right]_{+}
$$

If the number of unphysical states is different from zero

$$
\frac{\hat{N}}{N}\left|\varphi>_{a s}=\hat{Q}_{0}\right| \chi>
$$

where

$$
|\chi>=\hat{K}| \varphi>_{a s}
$$

Therefore any state annihilated by $\hat{Q}_{0}$ may be presented in the form

$$
|\varphi>=| \varphi>_{p h}+Q_{0} \mid \chi>
$$

where $\mid \varphi>_{p h}$ contains only three dimensionally transversal components of $A_{\mu}^{a}$ and the excitations corresponding to the complex doublet of the scalar mesons.

In the limit when the mass of the scalar mesons vanishes we have precisely the particle content described in the paper [2]. If the parameter $\mu$ is small, smaller than the accuracy of measurement, one cannot distinguish experimentally the transversal polarizations of the Yang-Mills field in these two scenarios, but the number of degrees of freedom is different. In the first case in the limit $\mu \rightarrow 0$ the longitudinal quanta decouple, whereas in the second case the charged scalar bosons interact with the massless Yang-Mills field even in the limit $\mu \rightarrow 0$.

\section{Conclusion.}

In this paper we showed that the three dimensionally longitudinal component of the vector field decouples of all other excitations in the massless limit of the nonabelian Higgs-Kibble model. This is in complete analogy with the Abelian case. The massless Yang-Mills theory interacting with massless scalar fields may be also obtained starting from the Yang-Mills field interacting with the complex scalar doublet of real mass, when this mass tends to zero. In this case the number of observable degrees of freedom is bigger: the charged scalar mesons of zero mass are also present.

\section{Acknowledgements.}

I wish to thank R.Ferrari for numerous discussions, which resulted in the appearance of this paper. This paper was supported in part by RFBR under grants 11-01-00296a and 11-01-12037 ofi-m-2011, by the grant of support of leading scientific schools NS-4612.2012.1 and by the program "Nonlinear dynamics".

\section{References}

[1] A.A.Slavnov, L.D.Faddeev, Theoretical and Mathematical Physics 03(1970)18. 
[2] R.Ferrari, arXiv:1106.5537.

[3] T.Kugo,I.Ojima,Suppl.Progr.Theor.Phys.(1979)N66. 